\title{
Adapting Management of Sarcomas in COVID-19: An Evidence-Based Review
}

\author{
Ashish Gulia ${ }^{1}$ (1) Ramandeep Singh Arora ${ }^{2}$. Pankaj Kumar Panda ${ }^{3} \cdot$ Anand Raja $^{4} \cdot$ Akshay Tiwari $^{5} \cdot$ Sameer Bakhshi $^{6}$. \\ Naveen Salins ${ }^{7} \cdot$ Vineeta Goel $^{8} \cdot$ Amit Janu $^{9}$
}

Received: 20 April 2020 / Accepted: 18 May 2020 / Published online: 30 May 2020

(C) Indian Orthopaedics Association 2020

\begin{abstract}
With the novel coronavirus disease (COVID-19) being declared a global pandemic by the World Health Organization, the Indian healthcare sector is at the forefront to deliver optimal care. Patients with cancer especially are at serious risk for increased chances of morbidity and mortality due to their immunocompromised state. Currently there is a paucity of definitive guidelines for the management of sarcomas during the pandemic in a resource-constrained and diverse population setting like India. Health care professionals from various specialties involved in the management of sarcomas have collaborated to discuss various aspects of evidence-based sarcoma management during the COVID-19 pandemic. This article provides structured recommendations for HCP to adapt to the situation, optimize treatment protocols with judicious use of all resources while providing evidence-based treatment for sarcoma patients.
\end{abstract}

Keywords Sarcoma $\cdot$ Treatment guidelines $\cdot$ COVID-19 $\cdot$ India

\section{Introduction}

The novel coronavirus disease (COVID-19) caused by the severe acute respiratory syndrome coronavirus 2

Ashish Gulia

aashishgulia@gmail.com

Ramandeep Singh Arora

childhoodcancer@gmail.com

Pankaj Kumar Panda

pankajpanda86@gmail.com

Anand Raja

dr_anand@yahoo.com

Akshay Tiwari

akshay_t_2000@yahoo.com

Sameer Bakhshi

sambakh@hotmail.com

Naveen Salins

naveen.salins@manipal.edu

Vineeta Goel

vineetagoel@yahoo.com

Amit Janu

amiteasy10@gmail.com

1 Bone and Soft Tissue Services, Department of Surgical Oncology, Tata Memorial Hospital, and Homi Bhabha National Institure (HBNI), Mumbai, India
(SARS-CoV-2) has been declared a global pandemic by the World Health Organization (WHO) on the 11th of March [1]. As of 17th April 2020, globally infections are crossing 2 million and there are over 150,000 fatalities [2]. In

2 Paediatric Oncology, Max Super Speciality Hospital, New Delhi, India

3 Department of Clinical Research, Apollo Proton Cancer Centre, Chennai, India

4 Department of Surgical Oncology, Cancer Institute (WIA), Adyar, Chennai, India

5 Musculoskeletal Oncology, Max Institute of Cancer Care, Saket, New Delhi, India

6 Department of Medical Oncology, Dr. B. R. A. Institute Rotary Cancer Hospital, All India Institute of Medical Sciences (AIIMS), New Delhi, India

7 Department of Palliative Medicine and Supportive Care, Kasturba Medical College, MAHE, Manipal, India

8 Radiation Oncology, Max Super Speciality Hospital, Shalimar Bagh, New Delhi, India

9 Department of Radiology, Tata Memorial Hospital, and Homi Bhabha National Institure (HBNI), Mumbai, India 
India, with a population of more than 1.3 billion, the number infected is above 15,000 and there have been $500+$ deaths [3]. With the rising number of infections and mortality in India the healthcare sector has to be at the forefront to deliver optimal care.

Most infected people develop mild respiratory symptoms, with around $20-30 \%$ of persons aged $\geq 45$ years requiring hospital admissions. The case fatality rate (CFR) across all countries affected has been 3.4\% [2]. Those with cancer or receiving treatment for cancer are at enhanced risk of serious morbidity, including the need for ventilator support or increased risk for mortality. This is because of their immunocompromised state caused not only by the malignancy but also by the anti-cancer therapies [4]. Consequent to the above observations, professional groups, and national organisations have come out with recommendations to delay treatment and reduce its intensity where it is possible [5, 6] and algorithms to stratify patients have been proposed $[7,8]$.

Currently there is a paucity of data and guidelines in managing musculoskeletal tumors during the COVID-19 pandemic which are wither too general [9] or brief $[10,11]$ and do not reflect the situation in resource-constrained settings like India. We aim to provide guidance to healthcare professionals involved in the management of sarcomas to adapt to the situation, optimise treatment protocols with judicious use of all resources like manpower, operation theatres, intensive care units, essential drugs, blood products while maintaining highest standards of evidence-based treatment.

\section{Challenges Specific to Sarcoma Patients}

Sarcomas are heterogeneous and are remarkable for their broad spectrum of clinical behaviour, from the slow-growing indolent tumours to the rapidly growing disseminated tumours. If treated timely and adequately, sarcomas have good oncological outcomes which are better than many other cancers [12]. The small risk of acquiring the SARS-CoV-2 infection has to be balanced against the need to treat the malignancy. If death, from SARS-CoV-2 while on treatment is undesirable, surviving SARS-CoV-2 and dying of untreated, delayed, or de-intensified treatment is worse. Identifying patients who may not have detrimental outcomes with delay in treatment vs patients who may have an inferior survival with delay is challenging.

Unique challenges [13]:

1. Biological aggressiveness does not always correlate with clinical outcomes.

2. Delay in the initiation of treatment correlates with inferior oncological outcomes.
3. Surgery places the patient for a longer time in the healthcare system increasing the risk of infection and adverse outcomes.

4. Many surgeries are complex, requiring blood transfusions, free flaps with increased operating time, postoperative recovery, and attendant complications.

5. Efforts to delay surgery by neo-adjuvant/adjuvant therapy may lead to immunocompromised status.

6. The role of chemotherapy in many sarcomas is controversial and risk/benefit has to be assessed.

7. Stopping treatment mid-way may lead to loss of dose intensity and inferior survival.

8. Patients with progression on neo-adjuvant therapy represent a unique subset who needs emergent surgery.

9. Unknown impact of "therapy holidays".

We need to modify sarcoma management practices during the COVID-19 pandemic to prevent additional infection of health care workers, patients, and their relatives. Hospitals can act as hotspots and be sources of very high transmission rates. Various steps should be taken at multiple levels during a patient's journey in the hospital to minimize transmission. In addition to practicing social distancing by minimizing patients and relatives in OPD and wards, it is advisable to spread the awareness regarding prevention of COVID-19 spread. Sarcoma management should be multidisciplinary even in these times. The treatment has to be individualised based on the clinical presentation, available resources, and after due discussions in the multi-disciplinary team (MDT) meetings. Multidisciplinary joint clinics must shift from a close room meeting to an interactive online meeting.

\section{Managing Patient Contact-Outpatient, Inpatient, Emergencies and Surgeries}

The outpatient clinic (OPD) is the first point of contact of cancer patients with HCWs. Reducing the number of people here is of paramount significance in reducing the spread of this infection. The strategies employed can be broadly classified as.

\section{Scheduling OPD Appointments}

- OPD visits of patients for second consultations, on longterm follow-up or those in routine surveillance after curative treatment as well as those being seen for supportive care-type visits like survivorship can be contacted and rescheduled after the COVID-19 crisis. Patients with 
specific concerns can be scheduled through teleconsultations.

- Patients under evaluation to have case records evaluated by treating team and deferred where delay in initiation of treatment might not lead to adverse oncological outcomes.

\section{Priority Stratification [14]}

- To have barriers during the interaction between patients and HCW. Provision for adequate hand sanitizers and signage to be available.

- Screening questions to be asked by HCW to include, history of foreign travel by self or close relative, history of contact with suspected or confirmed patient with COVID-19, history of respiratory symptoms or fever.

- Patients deemed at triage to be non-emergent to have a rescheduling of OPD visits.

\section{Precautions/Requirements for Patients}

- To come alone or a maximum of one attendant.

- Social distancing in OPD waiting halls.

- Patients to bring all copies of treatment including slides/ blocks of biopsies done elsewhere to minimize travel.

- Use of face masks.

- Cover any open wounds/raw areas with bandaged dressings as SARS CoV-2 has been detected in the blood.

\section{Precautions/Requirements for HCWs}

- The health care team treating patients has to be segregated into discrete units (depending on manpower), with no contact between units, and the units should manage the OPDs alternately. This strategy ensures continuity of care in the event of infection of any-one unit.

- HCWs should adhere to standard hygiene and universal safety precautions when caring for patients.

- Personal Protective Equipment (PPE) are protective gears designed to safeguard HCWs by minimizing exposure. Components include goggles, face-shield, mask (simple double layer cloth masks, Surgical 3-ply masks, N-95 Respirator mask), gloves, coverall/gowns, head and shoe cover. The intensity of PPE usage depends on risk PPEs are a precious commodity and must be used judiciously. Special care should be given to the use of N-95 face mask. These are in limited quantity and various techniques have been described for reuse [15].

\section{Infection Control [16]}

\section{For All Patients Suspected to Have COVID-19}

- Attendants should be strictly instructed to be beside the patient only.

- Patient to be referred to the designated testing centre, results to be followed up.

- Equipment used (if any) to be discarded in designated areas.

- Holding area preferable to be decontaminated.

\section{COVID 19 Testing and PPE [17]}

(a) Asymptomatic patients (No history of fever, cough, sore throat, olfactory dysfunction or travel history or history of contact with a suspected or proven case of COVID patient) may not require COVID-19 testing during OPD and follow up visits.

(b) Routine pre-operative testing for COVID-19 should be done for all patients undergoing any kind of surgical procedure.

(c) PPEs to be used in OPD and wards-3 ply surgical mask + face shield cloth gown + double gloves.

(d) For routine surgical procedures PPE should include routine standard PPE.

(e) $\mathrm{N} 95$ mask is recommended for procedures involving drilling, burring or use of saw, which are aerosol-generating. Minimum staff should be present in the OT.

(f) Special care must be taken to train HCWs in donning and doffing PPEs. Disposal of PPEs must be done in appropriate manners.

(g) A census of the other patients and staff people in the practice at the time of the patient visit is logged, so later they may be contacted if the patient turns out to be positive for COVID-19. If the patient is already positive, the room is cleaned and disinfected according to hospital protocols, including a 1-h downtime for passive air exchange.

\section{Diagnostics (Radiology/Nuclear Medicine/ Histopathology/Molecular Biology)}

Imaging in sarcoma forms part of initial staging workup, management and follow up. This begins with radiographs, followed by magnetic resonance imaging (MRI) for local staging, CT and bone scan/PET CT scan for metastasis. Diagnostic and staging workup must be done immediately to expedite treatment initiation. Three-dimensional imaging may be deferred in indolent benign/low-grade lesions if radiographs are diagnostic. Mandatory precautions 
must be taken while performing these studies. During the pandemic, nuclear imaging studies may not be available due to a lack of nuclear active isotopes. This can be deferred until the availability or alternative methods (Whole-body MRI) may be utilized if necessary.

All referrals for radiology in these times have to be decided by guidelines recommended by the infectious disease committee of the hospital after evaluation by MDT to decrease footfall in the radiology department. Long term follow-up may be delayed and if required can be done by centres closer to patients' residence to avoid travel and to reduce footfall in hospitals

RT-PCR tests are diagnostic to test for COVID as per WHO [18-20]. Advanced imaging should be done after a negative RT-PCR. In the absence of RT-PCR, CT chest with characteristic findings of SARS-CoV-2 infection can be used as an alternative.

CT suit decontamination required after scanning of every suspected/ positive COVID-19 patients will disrupt the radiological service availability hence many guidelines suggest portable chest $\mathrm{x}$-ray (CXR) to minimize the risk of cross-infection [21]. CXR findings have lower sensitivity than RT-PCR testing (69\% versus $91 \%$, respectively) and CT scans [22]. Portable X-ray units can be used in fever OPD and isolation wards. The consistent findings seen are peripheral, bilateral, lower zone pneumonitis, opacities, and patches without pleural effusions [23].

The focus of most radiology departments is shifting from diagnostic capability to preparedness. If investigations are needed and the patient is suspected case of COVID-19, HCWs should use appropriate PPE.

Histopathology should be expedited. Emphasis should on reevaluating previous biopsies to decrease repeat procedures. Biopsies must be performed by senior clinicians to avoid inconclusive reports. Tumor board meetings and discussions can be done telephonically, on WhatsApp groups or in video conference calls. The department should be able to work from home and give teleradiology and telepathology reports.

\section{Management of Sarcomas}

\section{Surgical Therapy}

Surgical management forms the cornerstone of treatment of bone and soft tissue sarcomas and is considered a semiemergency; the timing of surgery having important implications in the outcome of these patients. This underlying fact remains unchanged in COVID 19 pandemic era, particularly for high-grade bone and soft tissue sarcomas [11]. In the presence of COVID 19 pandemic, with the looming uncertainty of how the pandemic unfolds during the coming months, there are three major concerns that surround decisions regarding surgery for bone and soft tissue sarcomas-

- Higher risk of postoperative morbidity and mortality in COVID 19 positive patients [24], more so in cancer patients [4].

- Protection of healthcare workers from potential COVID19 infection from patients undergoing surgery.

- Utilization of precious resources (e.g. ICU beds/blood and blood products/PPEs), which may be needed, should there be a steep rise in other COVID-19 patients requiring hospitalization.

In the light of these considerations, the following may be a prudent outline for surgery for bone and soft tissue sarcomas.

\section{General Principles}

Curative surgery for high-grade bone and soft tissue sarcoma should proceed as planned.

- Adequate PPE and surgical and anesthesia consideration should be followed to minimize the risk of COVID 19 transmission [25].

- Curative surgery for locally aggressive bone lesions and low-grade sarcomas may be delayed.

- In an MDT setting, it is acceptable to avoid/defer major surgeries to a later suitable date provided the oncological outcome of a patient is not affected (e.g. continuing neo-adjuvant chemotherapy to delay internal hemipelvectomy in pelvic osteosarcoma responding well to chemotherapy).

- Palliative surgeries for sarcomas should be avoided during COVID-19 pandemic and alternative noninvasive options must be explored.

- For sarcomas with metastases, metastasectomy should be carefully considered on a case to case basis in curative settings (keeping number and site of metastases in mind), only after completion of chemotherapy wherever applicable.

- Special care must be taken for the management of sarcoma in elderly individuals with associated comorbidity.

- In proven/ highly suspected COVID positive patients all sarcoma treatment including surgery should be delayed until 15 days or recovery from symptoms/ patient turning seronegative, whichever is later.

\section{Soft Tissue Sarcomas (STS)}

- A primary high-grade soft tissue sarcoma without metastasis should be prioritized for surgery. 
- Resection of low-grade soft tissue tumors or those with a known indolent behavior/low metastatic risk (e.g. atypical lipomatous tumor, classic dermatofibrosarcoma protuberans, desmoid tumors, retroperitoneal well-differentiated liposarcoma, myxoid liposarcoma, low gradefibromyxoid tumor) can be deferred depending on available resources and logistics.

- Consider preoperative radiotherapy for large border-line resectable sarcomas.

- Complex surgical resections requiring additional bone/ vascular/nerve reconstruction or procedures requiring free flaps should be avoided.

\section{Bone Sarcomas/Tumors}

- Timely local therapy of malignant bone tumors should be done with or without modification in surgical techniques-more so with tumors showing poor response to neoadjuvant chemotherapy, large/ fungating tumors needing amputation.

- Surgical management of benign bone tumors or locally aggressive lesions can be delayed if deemed appropriate. Wherever applicable, percutaneous injection techniques like sclerotherapy/radiofrequency ablation should be preferred.

- Simpler and quick surgical reconstruction should be preferred.

- Patients having peri-prosthetic infections to be assessed on a case-by-case basis. In immunocompromised patients with severe/acute periprosthetic infections, wound wash and debridement should be considered. Mild/chronic infections should be managed conservatively.

- For Ewing's Sarcoma, surgery vs radiotherapy as local therapy to be decided on an individual case basis. Radiation to be considered as an alternative to surgeries, which are extensive/long duration/needing major blood transfusion/prolonged hospital stay.

\section{Chemotherapy}

\section{General Principles}

Chemotherapy is vital in optimizing outcomes including that in the neo-adjuvant setting for Ewing's sarcoma, osteosarcoma, and rhabdomyosarcoma. The use of neoadjuvant chemotherapy for high-grade sarcomas or recurrent sarcomas can be considered if it can be safely delivered as a means of deferring surgical intervention.

During this COVID-19 pandemic, an adaptation of chemotherapy may be necessary to ensure optimal outcomes while reducing the risk of SARS-CoV-2 infection to the child, family and HCWs by minimizing time spent in the hospital. It is important to highlight that the extent of

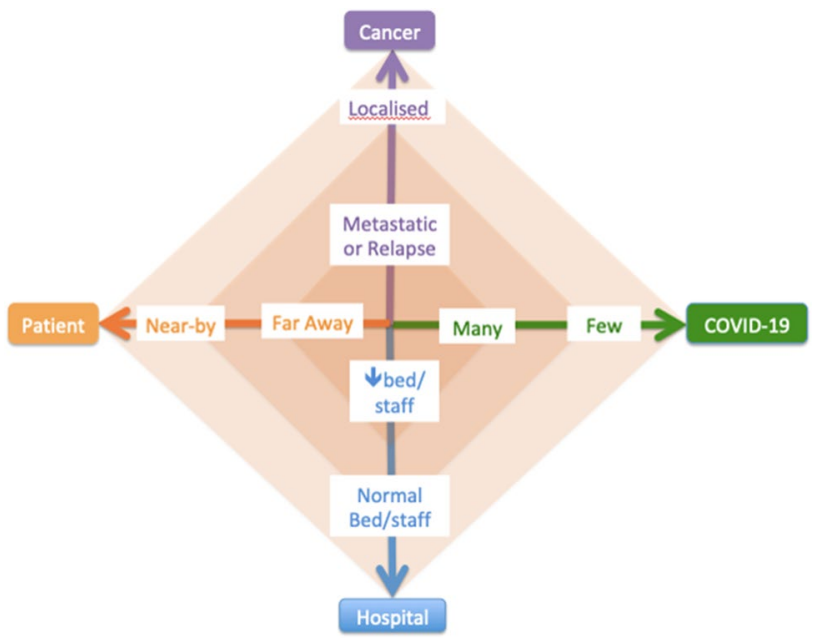

Fig. 1 Factors impacting the decision to adapt treatment with MAXIMUM adaptation at the center of the diamond and MINIMUM adaptation at the PERIPHERY of the diamond

adaptation is dependent on the patient's access to healthcare, extent and state of disease, resources in hospital and severity of the COVID-19 spread in the geography (Fig. 1). The adaptation can be achieved in several different ways:

- Use shorter regimens to avoid a hospital stay.

- Reducing the days to give the same chemotherapy in each cycle.

- Increasing the interval of chemotherapy between cycles.

- Decreasing the dose (reducing the risk of the need for blood products and febrile neutropenia) and hence inpatient admissions.

- Liberal use of Granular Colony Stimulating Factor (GCSF), particularly in the pegylated form.

- Consider deferring local control (surgery and or radiation therapy) by giving additional chemotherapy.

\section{Ewing Sarcoma, Osteosarcoma and Rhabdomyosarcoma}

The above sarcomas are seen predominantly in children, teenagers and young adults. Based on the above principles adaptations for the first-line treatment for patients with non-metastatic Ewing sarcoma, Osteosarcoma and Rhabdomyosarcoma during COVID-19 pandemic are suggested in Table 1.

\section{Metastatic Setting}

Ewing Sarcoma At Presentation: Initiate VAC,

Midway (intent palliative): Drug break acceptable as overall outcome is $<15 \%$ [31] and risk of acquiring COVID and transmitting may be more. 
Table 1 Suggested adaptations to chemotherapy in COVID-19 pandemic for non-metastatic Ewing sarcoma, Osteosarcoma and Rhabdomyosarcoma

\begin{tabular}{|c|c|c|}
\hline Cancer & Conventional first-line protocol & Suggested adaptations during COVID-19 pandemic \\
\hline Ewing Sarcoma & $\begin{array}{l}\text { Vcr, Dox, Cyclo (VDC)/Ifos, Etop (IE) every } \\
2 \text { or } 3 \text { weeks } \\
\text { Alternatives: Vcr, Ifos, Dox, Etop (VIDE) } \\
\text { followed by VAC or VAI }\end{array}$ & $\begin{array}{l}\text { Switching to } 3 \text { weekly schedules [26] } \\
\text { Replace multiple daily GCSF with a single dose of PEG-GCSF } \\
\text { Doxorubicin-infuse over } 6 \mathrm{~h} \text { in one day ( } 75 \mathrm{mg} / \mathrm{m}^{2} / \mathrm{day} \text { ) [27] } \\
\text { VAC instead of VAI is using Euro-Ewing protocol } \\
\text { Additional chemotherapy can be given as local control can be delayed up } \\
\text { to } 15 \text { weeks from the start of treatment [28] } \\
\text { Consider } 25 \% \text { dose reduction if needed }\end{array}$ \\
\hline Osteosarcoma & $\begin{array}{l}\text { Two drugs: Cispl, Dox (AP) } \\
\text { Three drugs: Ifos, Cispl, Dox (IAP); HDMtx, } \\
\text { Cispl, Dox (MAP) }\end{array}$ & $\begin{array}{l}\text { Switching to two drugs [29] } \\
\text { Doxorubicin-Infuse over } 6 \mathrm{~h} \text { in one day }\left(75 \mathrm{mg} / \mathrm{m}^{2} / \text { day) or } 2 \text { days }\right. \\
\left(37.5 \mathrm{mg} / \mathrm{m}^{2} / \text { day) rather than } 48-\mathrm{h} \text { infusion }[26]\right. \\
\text { Cisplatin can be given in two days }\left(50-60 \mathrm{mg} / \mathrm{m}^{2} / \text { day }\right) \\
\text { Consider IAP over MAP fro three-drug regimen as it reduces the fre- } \\
\text { quency of chemotherapy and days in the hospital } \\
\text { Use PEG-GCSF after each cycle } \\
\text { Consider } 25 \% \text { dose reduction if needed }\end{array}$ \\
\hline Rhabdomyosarcoma & $\begin{array}{l}\text { Vcr, Act D, Cyclo (VAC) } \\
\text { Alternatives: VAC/Vcr, Irin (VI); Vcr, Act D, } \\
\text { Ifos (VAI) can be the other alternatives }\end{array}$ & $\begin{array}{l}\text { Use VAC to reduce days in the hospital } \\
\text { Omit weekly Vcr } \\
\text { Additional Doxorubicin for high risk not of benefit [30] } \\
\text { Use PEG-GCSF after each cycle } \\
\text { Consider } 25 \% \text { dose reduction if needed }\end{array}$ \\
\hline
\end{tabular}

Osteosarcoma At initiation,

If metastasis $>3$, intent palliative hence chemotherapy may be deferred for few weeks [32].

If metastasis $<3$, treat as for non-metastatic osteosarcoma, Midway,

If metastasis persistent and intent palliative, defer chemotherapy,

If metastasis controlled, continue treatment as per protocol.

\section{Other Soft Tissue Sarcomas}

Neoadjuvant Setting Surgical excision alone is sufficient for patients with small $(<5 \mathrm{~cm})$, low-grade, and superficial (sarcomas, there is no role of neoadjuvant chemotherapy (NACT). In larger $(>5 \mathrm{~cm})$, high grade tumours also, the role of NACT is limited [33, 34]. Due to questionable activity, NACT may be avoided in localized soft tissue sarcomas during this COVID-19 pandemic.

Adjuvant Setting For patients who have undergone surgical resection, adjuvant chemotherapy may be delayed by a few weeks as the role of adjuvant chemotherapy is controversial [35]. If adjuvant RT is required and feasible, it is to be administered prior to adjuvant chemotherapy.

Metastatic Disease Asymptomatic Patients Standard chemotherapy with doxorubicin may be delayed by a few weeks keeping in mind the risk-benefit ratio of repeated hospital visits. If the expected delay is longer than 6-8 weeks, oral
Pazopanib may be initiated. For patients with progression post previous doxorubicin, oral therapy with pazopanib may be preferred due to good tolerability and activity [36]. For rare histologies like alveolar soft part sarcoma, solitary fibrous tumour, and clear cell sarcoma, sunitinib may be used [37-39].

Symptomatic Patients Reduced dose single-agent doxorubicin with pegfilgrastim may be used [40]; reduced doses with liberal use of growth factors and especially single dose pegylated forms may be used to reduce hospital visits and limit toxicity related visits. It must be kept in mind that reduced doses are unlikely to compromise curability and the intent in such situations is palliative. Once symptoms improve intravenous doxorubicin may be changed to oral Pazopanib to avoid frequent hospital visits.

Progression Post Doxorubicin/Pazopanib Intravenous agents such as Trabectidin, a combination of gemcitabine and docetaxel, and eribulin are known to be beneficial in these situations. As overall intent is palliative, and the difficulty and risk involved in frequent hospital visits appears to be greater than the modest benefit hence we believe it is reasonable to delay use of these agents in the current COVID19 pandemic situation.

Oral metronomic therapy (a combination of thalidomide, celecoxib, cyclophosphamide and etoposide) has been found useful in non-bone sarcoma solid tumors with minimal toxicity $[41,42]$, this may be a reasonable option in this 
pandemic situation in patients who have progressed beyond two lines of therapy.

\section{Radiation Therapy $[11,43,44]$}

If there is an indication for radiation therapy, plan to do it preoperatively in order to delay the timing of surgery for about 3-4 months. Also, consider the use of preoperative radiation therapy as a bridge therapy to postpone surgery when appropriate, even if the treatment is not standard, but there is evidence that it won't harm (i.e. preoperative radiation therapy in retroperitoneal liposarcoma).

\section{High Priority (Continue Radiation Therapy as Planned in Non-COVID Times)}

Any patient being treated with curative intent and needs neoadjuvant/adjuvant/radical RT and where delay in radiation therapy is likely to decrease cure rates should be treated with highest priority.

\section{High-Grade STS}

- Whenever feasible do intraoperative interstitial brachytherapy as it provides the shortest course of treatment.

- Strongly consider neo-adjuvant radiation therapy if sarcoma is unresectable, surgery not feasible due to the COVID pandemic.

- Post-operative adjuvant radiation therapy may be delayed by $6-8$ weeks from the date of surgery.

2. Ewings Sarcoma

- Consider radical radiation therapy in place of surgery for patients where either disease is unresectable or surgery is not possible due to COVID-19 pandemic (eg. limb salvage surgery with suitable prosthesis).

- If Ewing's Sarcoma is resectable, however surgery is not possible at the correct time, one may consider pre-operative RT.

3. Rhabdomyosarcoma-If planned for definitive radiation therapy, continue radiation therapy as per protocol without delays/deviations.

4. Palliative Radiation Therapy- Patients with metastatic diseases and with cord compression/bleeding/dysphagia

\section{Intermediate Priority}

- Post-operative radiation therapy in Ewings Sarcoma/ Rhabdomyosarcoma
Low Priority- (RT for Slowly Proliferative Tumors/ Prophylactic RT Can be Deferred)

- Low-grade soft tissue sarcoma requiring postoperative radiation therapy.

- Fibromatosis.

- Lung Bath for prophylaxis in sarcomas.

- SBRT for asymptomatic lung metastases from sarcoma.

- Chordomas/chondrosarcoma-radical/postoperative radiation therapy.

\section{Recommendations for Radiation Therapy}

- Any patient who has started radiation therapy must continue treatment.

- Avoid protracted 6-7 weeks course of treatment. Use suitably hypofractionated regimen of dose fractionation calculated using LQ model and in consideration of surrounding normal structures.

- Radiotherapy interruptions if any should be managed as per standard guidelines.

- Pediatric patients requiring daily radiation therapy under anesthesia is certainly more challenging and needs to be decided based on the availability of local resources. As far as a possible treatment of pediatric patients should be carried out as per protocol.

\section{Precautions and Use of PPE During Radiation Therapy Planning and Treatment}

- As per present ICMR guidelines, only symptomatic patients or patients with a history of contact with COVID-19 positive cases, need to be tested before starting radiation therapy.

- All staff members in the radiation therapy unit need to take universal safety precautions like the use of basic PPE. Frequent hand washing, sanitization, cleaning of Linac couch and immobilization devices with hypochlorite solutions should also be carried out.

\section{Psychosocial Support/Care}

There are multiple sources for emotional distress in patients and families during the COVID-19 pandemic [45]. The pandemic per se can cause distress due to social isolation, anxiety, depression and delirium. A study assessing the psychological impact of the COVID-19 outbreak showed that one-third of the patients develop moderate to severe anxiety symptoms and one-sixth of the patients develop moderate to severe depressive symptoms [46]. Some families may be barred from the community due to illness and develop 
negative perceptions about their illness due to stigmatisation, authoritarianism and poverty.

Distress in patients with cancer can get compounded in a pandemic situation due to disruption in the cancer treatment, increased risk of cancer progression, and a higher risk of contracting COVID-19 in the hospital due to their immunosuppressive state [47]. The uncertainty about initiating, continuation and receiving cancer treatment and unpredictability of the course of cancer in these circumstances can be distressing leading to unresolved emotional issues.

To accommodate infection control and social distancing practices during the COVID-19 pandemic traditionally provided face to face psychosocial support as an individual or group therapy needs to be altered and it may have to be offered virtually via telephone or video chat by adequately trained frontline $\mathrm{HCW}$.

Similarly, counseling support, (which involves continual reassurance, positive thoughts, coping strategies, and helping patients and families to navigate the maze of information and misinformation) and pharmacological management for depression and anxiety can be provided by frontline health workers under the telephonic guidance of mental health professionals [48].

Face to face or telephonic communication with patients and families is critical. The communication should be positive and bidirectional and should not create more anxiety or fear either due to COVID 19 pandemic or disease.

\section{Palliative Care}

Patients receiving palliative intent treatment are less likely to receive cancer treatment during the COVID-19 pandemic. Recently a conceptual framework for prioritization of radiotherapy and systemic treatment during COVID-19 showed that palliative intent therapy with modest or no survival benefit and modest or no symptom benefit are categorized as low priority cancer treatment [8]. The patients with advanced sarcoma can have pain due to local disease or skeletal metastasis. Breathlessness is often seen due to lung metastasis [49]. Management of breathlessness and pain in patients with advanced sarcomas are provided in Algorithm 1 and 2 (Figs. 2, 3) [50-54].

In patients with advanced sarcoma, it may be useful to discuss the goals of care and plan for treatment. The primary purpose of advance care planning is to avoid intensive care admissions in patients who are unlikely to benefit from ICU measures and ventilation. In the current pandemic situation, invasive ventilation and admissions in the hospital must be avoided. The patients who are terminally ill and needing end of life care management should be promptly referred to palliative care for further management [55-57] and should be managed at home as much as possible.

\section{Follow-Up}

Telemedicine has emerged as an important way of communicating with patients in the current COVID 19 outbreak. Ministry of health, Government of India has also accepted telemedicine as an alternative to outpatient consultation in the COVID-19 pandemic scenario to avoid direct contact between patient and physician and maintain social distancing. This has also been helpful in decreasing anxiety among oncology patients. Standard operating procedures (SOP) for teleconsultation in sarcoma follow up patients are:

- The teleconsultation calls need to be made by a doctor or a trained healthcare professional.

- During the teleconsultation, patients should be questioned about their general health status, any signs and symptoms of recurrent disease.

- Patients to be asked to do X ray of local site and CXR if required based on histology. Each patient should receive a contact number and an email address for further communication.

\section{Clinical Trials}

Along with the routine care of patients with sarcomas during the COVID-19 pandemic being impacted, challenges in conducting clinical trials and research have also come up with the evolving situation. This calls for the need to develop pragmatic guidelines to conduct clinical trials/ research ensuring the rights safety and well-being of the sarcoma patients.

\section{Initiating New Trials/Studies [58]}

- Targeted clinical studies/trials can be initiated during this challenging pandemic situation to study the impact of a pandemic or adverse situations on the oncologic and psychosocial outcomes of sarcomas in not only the patients but also the impact on their caregivers. The ethics committee and regulatory authorities should expedite the approval of these studies to be conducted and add to the advancement of sarcoma management principles.

\section{Impact of COVID-19 Pandemic on the Conduct of Ongoing Sarcoma Trials $[59,60]$}

- Challenges such as difficulty in visiting healthcare facilities for the sarcoma trial participants and trial staff will impact medical oversight and could adversely impact activities related to the conduct of trials such as completion of study assessments, study visits and the availability 
Algorithm 1: Management of Breathlessness in Advanced Sarcoma Patients

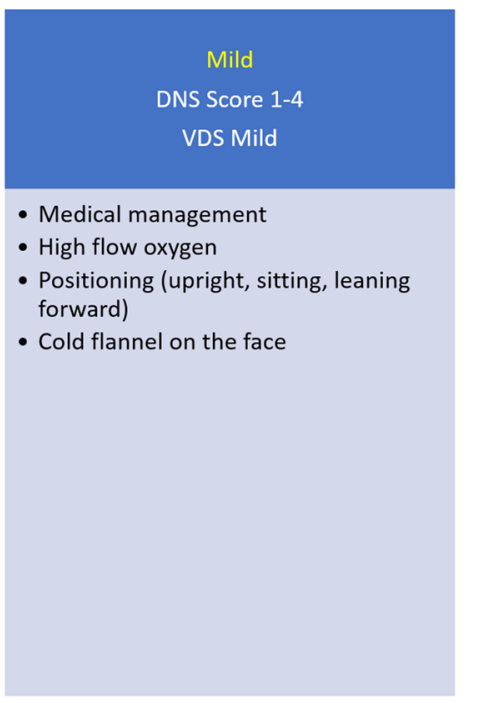

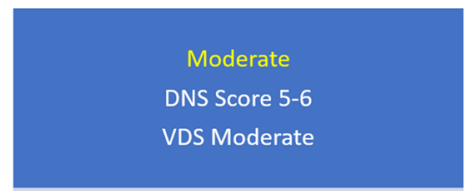

- Strategies used for Mild +

- Oral Morphine Immediate Release 2.5 $\mathrm{mg}$ BD-TDS $+2.5 \mathrm{mg}$ SOS. Slow upward titration by $2.5 \mathrm{mg}$ daily up to $40-60 \mathrm{mg} /$ day. If pain is present titration according to pain scores/pain relief

- Oral Lorazepam $0.5 \mathrm{mg}$ if anxiety is present. Increase by $0.5 \mathrm{mg}$ daily up to $4 \mathrm{mg} /$ day.

- Tab Metoclopramide $10 \mathrm{mg}$ SOS for nausea \& vomiting

- Tab Bisacodyl 10mg HS

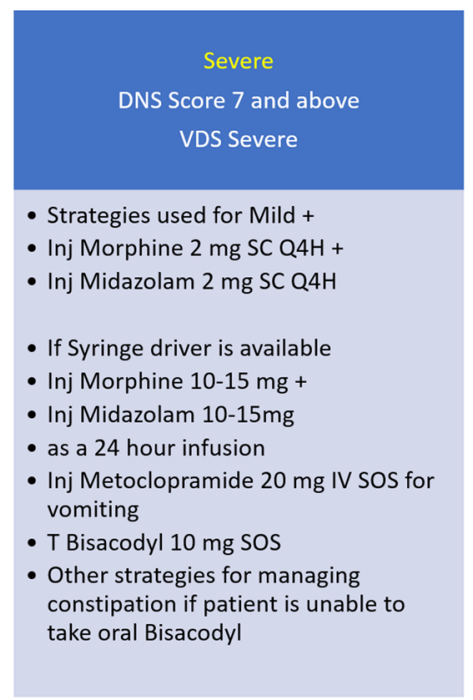

DNS = Dyspnoea Numerical Scale, VDS= Verbal Descriptor Scale for Dyspnoea

Fig. 2 Algorithm 1: management of breathlessness in advanced sarcoma patients

Algorithm 2: Management of Pain in Advanced Sarcoma Patients
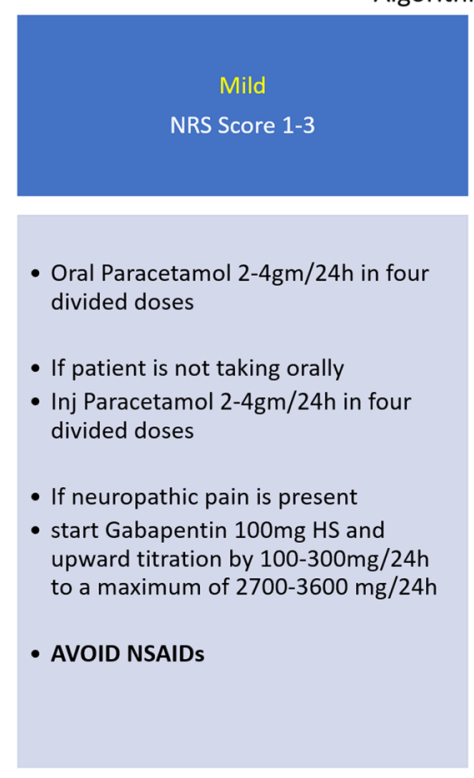

NRS $=$ Numerical Rating Scale for Pain

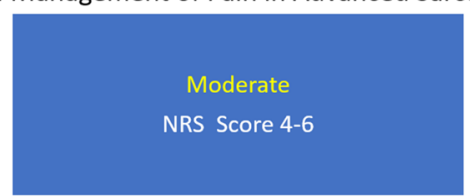

- Strategies used for Mild +

- Oral Morphine Immediate Release $5 \mathrm{mg} \mathrm{Q} 4 \mathrm{H}$ and breakthrough dose is $1 / 6^{\text {th }}$ the 24 hour dose. Upward titration by $50 \%$ of dose everyday

- If patient unable to take orally Inj Morphine $1-2 \mathrm{mg} \mathrm{SC}$ or IV every 4 hours

- Consider Fentanyl if patient has renal failure. Fentanyl dose is $0.2-$ $0.5 \mathrm{mcg} / \mathrm{kg} / \mathrm{hr}$

- Tab/Inj Metoclopramide 10-20 mg SOS for nausea \& vomiting

- Tab Bisacodyl 10mg HS

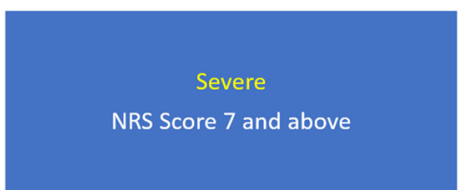

- Strategies used for Mild +

- Inj Morphine 2-2.5 mg SC Q4H

- If Syringe driver is available

- Inj Morphine 10-15 mg +

- as a 24 hour infusion

- Consider Fentanyl if patient has renal failure. Fentanyl dose is $\mathbf{0 . 2 -}$

$0.5 \mathrm{mcg} / \mathrm{kg} / \mathrm{hr}$

- Inj Metoclopramide 20 mg IV SOS for vomiting

- T Bisacodyl $10 \mathrm{mg}$ SOS

- Other strategies for managing constipation if patient is unable to take oral Bisacodyl

Fig. 3 Algorithm 2: management of pain in advanced sarcoma patients

of investigational drugs or materials/prostheses. Where required, recruitment should be temporarily halted, or suspended and subjects discontinued with special consideration to immunocompromised patients, subjects over 60 years of age or having debilitating medical conditions.

- Telephonic or video calling contact establishment should be done or home nursing visits should be facilitated to ensure continued medical oversight.
- An increase in study protocol violations and deviations may be noted during this time. All of these should be documented appropriately and reported to the ethics committee and suitable regulatory authorities as per applicable timelines and other guidelines.

- Suitable modifications in the study protocol, participant information documents and informed consent forms to appraise the patients of the increased risk during the 
pandemic should be made and must be approved by the ethics committee before any further study-related procedures.

\section{Medico-Legal Aspects}

During the COVID-19 pandemic, when to treat and when to wait is a conundrum. Medico-legally this is a grey area with a fair share of challenges. Some of the key challenges being a denial of treatment and its detrimental effects to cancer patients and the risk of contracting COVID-19 infection during hospitalization.

The best strategy for every hospital/ practitioner during this time to safeguard itself from medico-legal complications is to have a SOP which comprehensively covers every aspect of patient care from outpatients to inpatients to rescheduling of treatment to emergencies to surgeries to stop accepting new patients etc. and having HCWs adhere to the SOP. In addition, every patient that comes to the hospital should have clear documentation in the patient case records as to the plan of action and the reasons for the same, indexed to the SOP document. The same should be extended to all tele-consults and reschedules for treatment. Recording of tele-consults and having recorded MDT meetings involving decision making might mitigate the risk.

A detailed consent at presentation to the hospital and before a procedure is performed, informing the patient of the reasons for hospitalization and the risk of transmitting and contracting the infection (Figs. 4, 5) is essential.
In summary, communication with patients, a clear SOP, documentation of all actions in case records, detailed consents and recording of tele-consults and may mitigate the risk of medico-legal cases.

\section{Use of Modern Technology}

As with most other sectors, healthcare too is bracing up to the use of virtual communication tools. The present times pose an opportunity to explore video conferencing, webinars, virtual tumour boards and tele-consultations as powerful tools of communication between cancer patients, caregivers and centres [61]

Most centres have started teleconsultations/ virtual consult platforms, on easy to use apps, wherein reports/ images and the patients' narrative can be shared while allowing also for a real-time consultation. As long as both the doctors and the patients understand the limitations of virtual consults, they should be encouraged to use them, thereby reducing the travel and crowding of patients under follow up, under investigation, and waiting for treatment to begin.

Similarly, virtual tumor boards have now been in use for a long time, one example being the Virtual Tumor Board by the National Cancer Grid in India [62]. Such tumor boards should also be extensively used within and across centres, enabling multidisciplinary management while maintaining social distancing. The same platforms may be used for classes, webinars, grand rounds and other academic purposes.
Fig. 4 Sample declaration form at presentation to the hospital during COVID-19 pandemic
Name of the hospital

Doctor's Name

Date and Time

* Declaration - *

During the lockdown in the wake of the current Corona companion, I have come to the hospital by myself as an Emergency Treatment.

If I have an asymptomatic carrier or an undiagnosed patient with COVID19, I suspect it may endanger doctors and hospital staff, It is my responsibility to take appropriate precautions and to follow the Protocols prescribed by them.

I also know that I may get an infection from the hospital or from a doctor, and I will take every precaution to prevent this from happening, but I will Not at all hold doctors and hospital staff accountable if such infection occurs to me or my accompanying persons.

Patient Sign/Thumb Impression

Patient Name:

Mobile No:

Address: 
Fig. 5 Sample consent form before any surgical/anaesthesia procedures during COVID-19 pandemic

\section{COVID-19 SPECIFIC SURGICAL/ ANAESTHESIA CONSENT}

NAME:

AGE:

HOSPITALID:

\section{DIAGNOSIS:}

\section{PROCEDURE:}

1) Surgery is being planned for me as it is considered very essential and further delay will be oncologically unacceptable. This is being performed in the interest of patient and as there is no other significantly effective alternative modality of treatment available.

2) In view of the high infectivity rate of novel corona virus (COVID-19), I have been informed of the high chance of acquiring the infection during the procedure (surgery and anaesthesia) or during the postoperative stay in the hospital, in which case I will not hold the Institution/medical staff/paramedical staff/other hospital personnel etc, responsible for the adverse outcomes.

3) There are high chances of morbidity and mortality if a cancer patient acquires COVID19 infection in view of the immuno-compromised status and this can be further worsened by pre-existing co-morbid conditions if any.

4) In the eventl develop any fever, breathing difficulty, cough, in case of suspicion of COVID-19 infection, I have been explained that I may be referred to a Government Hospital/other centre for treatment in that scenario.

5) I am willing to undergo the surgery after understanding all the implications.

$\begin{array}{llc}\text { Patient Name } & \text { Patient Sign } & \text { Date } \\ \text { Attendant Name } & \text { Attendant Sign } & \text { Date } \\ \text { Doctor Name } & \text { Doctor Sign } & \text { Date }\end{array}$

\section{Compliance with Ethical Standards}

Conflict of interest The authors declare that they have no conflict of interest.

Ethical standard statement This article does not contain any studies with human or animal subjects performed by the any of the authors.

Informed consent For this type of study informed consent is not required.

\section{References}

1. https://www.who.int/emergencies/diseases/novel-coronaviru s-2019. Accessed on 18 Apr 2020

2. Worldometer. COVID-19 coronavirus pandemic. https://www. worldometers.info/coronavirus/. Accessed on 18 Apr 2020
3. https://www.covid19india.org/. Accessed on 18 Apr 2020

4. Liang, W., Guan, W., Chen, R., et al. (2020). Cancer patients in SARS-CoV-2 infection: a nationwide analysis in China. The lancet Oncology, 21, 335-337.

5. Burki, T. K. (2020). Cancer guidelines during the COVID-19 pandemic. The lancet Oncology, 21, 629-630.

6. You, B., Ravaud, A., Canivet, A., et al. (2020). The official French guidelines to protect patients with cancer against SARS-CoV-2 infection. Lancet Oncol

7. Al-Shamsi, HO., Alhazzani, W., Alhuraiji, A., et al. (2020). A practical approach to the management of cancer patients during the novel coronavirus disease 2019 (COVID-19) pandemic: an international collaborative group. Oncologist

8. Hanna, T. P., Evans, G. A., \& Booth, C. M. (2020). Cancer, COVID-19 and the precautionary principle: prioritizing treatment during a global pandemic. National Review Clinical Oncology, 17, 268.

9. https://sarcoma.org.uk/coronavirus-covid-19-advice. Accessed on 17th Apr 2020 
10. https://www.surgonc.org/wp-content/uploads/2020/03/SarcomaResource-during-COVID-19-3.30.20.pdf. Accessed on $17 \mathrm{Apr}$ 2020

11. Penel, N., Bonvalot, S., Minard, V., et al. (2020). French Sarcoma Group proposals for management of sarcoma patients during COVID-19 outbreak [published online ahead of print, $2020 \mathrm{Apr}$ 9]. Ann Oncol

12. Dancsok, A. R., Asleh-Aburaya, K., \& Nielsen, T. O. (2016). Advances in sarcoma diagnostics and treatment. Oncotarget, 8, 7068-7093.

13. Loong, H. H., Blay, J. Y., \& Munhoz, R. R. (2019). International collaborations and regional challenges in providing specialist multidisciplinary sarcoma care. American Society Clinical Oncology Educational Book, 39, 616-623.

14. https://www.vidanthealth.com/VidantHealth/media/Documents/ Outpatient-Triage-Assessment-Guidance-3-16-20-FINAL.pdf. Accessed on 17th Apr 2020

15. https://www.cdc.gov/coronavirus/2019-ncov/hcp/ppe-strategy/ decontamination-reuse-respirators.html. Accessed on 17th Apr 2020

16. Centre for disease control, COVID-19 for health professionals. https://www.cdc.gov/coronavirus/2019-ncov/hcp/faq.html. Accessed on 17th Apr 2020

17. https://icmr.nic.in/sites/default/files/upload_documents/Strat egey_for_COVID19_Test_v4_09042020.pdf Version 4. Accessed on 9th Apr 2020

18. Rubin, E. J., Baden, L. R., Morrissey, S., \& Campion, E. W. (2020). Medical journals and the 2019-nCoV outbreak. New England Journal of Medicine, 382(9), 866.

19. Corman, V. M., Landt, O., Kaiser, M., et al. (2020). Detection of 2019 novel coronavirus (2019-nCoV) by real-time RT-PCR. Eurosurveillance, 25(3), 2000045.

20. Long, C., Xu, H., Shen, Q., et al. (2020). Diagnosis of the coronavirus disease (COVID-19): rRT-PCR or CT? European Journal of Radiology, 25, 108961.

21. American College of Radiology (2020). ACR recommendations for the use of chest radiography and computed tomography (CT) for suspected COVID-19 infection. Updated 19 Mar 2020

22. Wong, H. Y., Lam, H. Y., Fong, A. H., et al. (2020). Frequency and distribution of chest radiographic findings in covid-19 positive patients. Radiology, 27, 201160.

23. Hansell, D. M., Bankier, A. A., MacMahon, H., et al. (2008). Glossary of terms for thoracic imaging. Fleischner Society. Radiology, 246, 697-711.

24. Lei, S., Jiang, F., Wating, S., et al. (2020). Clinical characteristics and outcomes of patients undergoing surgeries during the incubation period of COVID-19 infection. EClinicalMedicine, 5,100331

25. Viswanath, A., \& Monga, P. (2020). Working through the COVID-19 outbreak: Rapid review and recommendations for MSK and allied health personnel. Journal of Clinical Orthopaedics and Trauma. https://doi.org/10.1016/j.jcot.2020.03.014.

26. Womer, R. B., West, D. C., Krailo, M. D., et al. (2012). Randomized controlled trial of interval-compressed chemotherapy for the treatment of localized Ewing sarcoma: a report from the Children's Oncology Group. Journal of Clinical Oncology, 30(33), 4148-4154.

27. Loeffen, E. A. H., van Dalen, E. C., Mulder, R. L., \& Anthracycline Cardiotoxicity Working Group. (2018). The duration of anthracycline infusion should be at least one hour in children with cancer: a clinical practice guideline. Pediatric Blood Cancer, 65(2), e26867.

28. Lin, T. A., Ludmir, E. B., Liao, K. P., et al. (2019). Timing of local therapy affects survival in ewing sarcoma. International Journal of Radiation Oncology Biology Physics, 104(1), 127-136.
29. Anninga, J. K., Gelderblom, H., Fiocco, M., et al. (2011). Chemotherapeutic adjuvant treatment for osteosarcoma: where do we stand? European Journal of Cancer, 47(16), 2431-2445.

30. Bisogno, G., Jenney, M., Bergeron, C., \& European paediatric Soft tissue sarcoma Study Group. (2018). Addition of dose-intensified doxorubicin to standard chemotherapy for rhabdomyosarcoma (EpSSG RMS 2005): a multicentre, open-label, randomised controlled, phase 3 trial. The lancet Oncology, 19(8), 1061-1071.

31. Biswas, B., Rastogi, S., Khan, S. A., et al. (2014). Hypoalbuminaemia is an independent predictor of poor outcome in metastatic Ewing's sarcoma family of tumours: a single institutional experience of 150 cases treated with uniform chemotherapy protocol. Clinical Oncology (Royal College of Radiologists), 26(11), 722-729.

32. Nataraj, V., Rastogi, S., Khan, S. A., et al. (2016). Prognosticating metastatic osteosarcoma treated with uniform chemotherapy protocol without high dose methotrexate and delayed metastasectomy: a single center experience of 102 patients. Clinical and Translational Oncology, 18(9), 937-944.

33. Gortzak, E., Azzarelli, A., Buesa, J., et al. (2001). A randomised phase II study on neo-adjuvant chemotherapy for "high-risk" adult soft-tissue sarcoma. Eur J Cancer Oxf Engl 1990, 37(9), 1096-1103.

34. Pasquali, S., \& Gronchi, A. (2017). Neoadjuvant chemotherapy in soft tissue sarcomas: latest evidence and clinical implications. Ther Adv Med Oncol, 9(6), 415-429.

35. Sarcoma Meta-analysis Collaboration. (1997). Adjuvant chemotherapy for localised resectable soft-tissue sarcoma of adults: meta-analysis of individual data. Sarcoma Meta-analysis Collaboration. Lancet, 350(9092), 1647-1654.

36. van der Graaf, W. T. A., Blay, J.-Y., Chawla, S. P., et al. (2012). Pazopanib for metastatic soft-tissue sarcoma (PALETTE): a randomised, double-blind, placebo-controlled phase 3 trial. Lancet, 379(9829), 1879-1886.

37. Stacchiotti, S., Tamborini, E., Marrari, A., et al. (2009). Response to sunitinib malate in advanced alveolar soft part sarcoma. Clin Cancer Res Off J Am Assoc Cancer Res, 15(3), 1096-1104.

38. Stacchiotti, S., Negri, T., Libertini, M., et al. (2012). Sunitinib malate in solitary fibrous tumor (SFT). Ann Oncol Off $J$ Eur Soc Med Oncol, 23(12), 3171-3179.

39. Stacchiotti, S., Grosso, F., Negri, T., et al. (2010). Tumor response to sunitinib malate observed in clear-cell sarcoma. Ann Oncol Off J Eur Soc Med Oncol, 21(5), 1130-1131.

40. Edmonson, J. H., Ryan, L. M., Blum, R. H., et al. (1993). Randomized comparison of doxorubicin alone versus ifosfamide plus doxorubicin or mitomycin, doxorubicin, and cisplatin against advanced soft tissue sarcomas. Journal Clinical Oncology Offcial Journal American Society Clinic Oncology, 11(7), 1269-1275.

41. Pramanik, R., Agarwala, S., Gupta, Y. K., et al. (2017). Metronomic chemotherapy vs best supportive care in progressive pediatric solid malignant tumors: a randomized clinical trial. JAMA Oncology, 3(9), 1222.

42. Pramanik, R., \& Bakhshi, S. (2019). Metronomic therapy in pediatric oncology: a snapshot. Pediatric Blood Cancer, 66(9), e27811.

43. https://www.rcr.ac.uk/sites/default/files/paediatric-tya-patientsradiotherapy-covid19.pdf. Accessed on 17 Apr 2020

44. https://www.rcr.ac.uk/sites/default/files/sarcoma-treatment-covid 19.pdf. Accessed on 17 Apr 2020

45. World Health Organization (2018). Integrating palliative care and symptom relief into responses to humanitarian emergencies and crises: a WHO guide. 2018

46. Wang, C., Pan, R., Wan, X., et al. (2020). Immediate psychological responses and associated factors during the initial stage of the 2019 coronavirus disease (COVID-19) epidemic among the 
general population in china. International Journal of Environmental Research Public Health, 17(5), 1729.

47. Huremović, D. (2019). Psychiatry of pandemics: a mental health response to infection outbreak. Berlin: Springer.

48. Duan, L., \& Zhu, G. (2020). Psychological interventions for people affected by the COVID-19 epidemic. Lancet Psychiatry, 7(4), 300-302.

49. Gough N. J., Smith C., Ross J. R., Riley J. R., Riley J., Judson I. (2011). Symptoms burden, survival and palliative care in advanced soft tissue sarcoma. Sarcoma, 2011:8. https://doi. org/10.1155/2011/325189.325189

50. Day, M. (2020). Covid-19: ibuprofen should not be used for managing symptoms, say doctors and scientists. BMJ, 17(368), m1086.

51. Hajiesmaeili, M. R., \& Safari, S. (2012). Pain management in the intensive care unit: do we need special protocols? Anesthesiology Pain Medicine, 1(4), 237-238.

52. Pasero, C., Puntillo, K., Li, D., et al. (2009). Structured approaches to pain management in the ICU. Chest, 135(6), 1665-1672.

53. Davidson, P., \& Currow, D. (Eds.). (2010). Management of refractory dyspnoea: evidence-based interventions Cancer Forum. Sydney: Australian Cancer Society.

54. Rocker, G. M., Simpson, A. C., Horton, R., et al. (2013). Opioid therapy for refractory dyspnea in patients with advanced chronic obstructive pulmonary disease: patients' experiences and outcomes. CMAJ Open, 1(1), E27.

55. Borasio, G. D., Gamondi, C., Obrist, M., \& Jox, R. (2020). COVID-19: decision making and palliative care. Swiss Medical Weekly, 150(1314), 1.
56. Curtis, J. R., Downey, L., Back, A. L., et al. (2018). Effect of a patient and clinician communication-priming intervention on patient-reported goals-of-care discussions between patients with serious illness and clinicians: a randomized clinical trial. JAMA International Medicine, 178(7), 930-940.

57. You, J. J., Fowler, R. A., \& Heyland, D. K. (2014). Just ask: discussing goals of care with patients in hospital with serious illness. CMAJ, 186(6), 425-432.

58. https://www.fda.gov/drugs/coronavirus-covid-19-drugs/clinicaltrial-conduct-during-covid-19-pandemic. Accessed on $12 \mathrm{Apr}$ 2020

59. https://ec.europa.eu/health/sites/health/files/files/eudralex/vol10/ guidanceclinicaltrials_covid19_en.pdf. Accessed on 12 Apr 2020

60. https://www.hpra.ie/homepage/medicines/regulatory-informatio n/clinical-trials/covid-19-(coronavirus)-and-cts. Accessed on 12 Apr 2020

61. American Association for Cancer Research (2020). Cancer Care Goes Virtual in Response to COVID-19. Cancer Discov

62. Sengar, M. (2019). PSY2-8 - national cancer grid: a model for collaboration in cancer care and research. Annals of Oncology, 30 (Supplement 6), vi12.

Publisher's Note Springer Nature remains neutral with regard to jurisdictional claims in published maps and institutional affiliations. 